\section{PREVALENCIA E FATORES ASSOCIADOS AO SOBREPESO E OBESIDADE ENTRE ADOLESCENTES DE UMA ESCOLA PÚBLICA}

\author{
Prevalence and factors associated with overweight and obesity \\ among adolescents of a public school
}
Prevalencia y factores asociados con el sobrepeso y la obesidad de adolescentes de una escuela pública

\section{RESUMO}

Objetivo: Verificar a prevalência e os fatores associados ao sobrepeso e à obesidade entre adolescentes de uma escola pública em Campinas, São Paulo. Métodos: Estudo observacional, transversal, realizado entre julho e setembro de 2013, com 107 jovens entre 15 e 19 anos que cursavam o ensino médio em uma escola pública do município de Campinas, São Paulo. Utilizou-se um questionário para investigar dados sociodemográficos e fatores de risco para sobrepeso e obesidade. Além disso, verificaram-se dados clínicos (peso, altura, pressão arterial). Resultados: A amostra se caracterizou com predomínio de mulheres $(\mathrm{n}=65$, $60,7 \%$ ) com 16,5 anos em média e renda familiar entre 2 e 4 salários mínimos ( $\mathrm{n}=53,49,5 \%$ ). A prevalência de sobrepeso e obesidade foi de $18(16,8 \%)$ e $9(8,4 \%)$, respectivamente. Destaca-se que 62 (58\%) sempre omitiam uma refeição, 54 (50,5\%) sempre se alimentavam vendo televisão e $56(52,3 \%)$ não praticavam atividade física fora da escola. Tentar e não conseguir fazer dieta foi associado ao sobrepeso e à obesidade, e autoimagem curvilínea foi associada à obesidade. Conclusão: $\mathrm{O}$ estudo revelou que parte significativa da amostra estava com sobrepeso ou obesidade. A falha em manter uma dieta e a autoimagem curvilínea associadas às alterações nutricionais sugerem que os adolescentes tinham consciência dessas alterações e se preocupavam com o próprio peso, a ponto de buscarem a dieta para tentar emagrecer.

Descritores: Nutrição do Adolescente; Obesidade Pediátrica; Sobrepeso.

\section{ABSTRACT}

Objective: To determine the prevalence and factors associated with overweight and obesity among adolescents of a public school in Campinas, São Paulo. Methods: Observational, cross-sectional study conducted between July and September 2013 with 107 young individuals aged 14-19 years who attended high school in a public school in the municipality of Campinas, São Paulo. It was used a questionnaire to investigate sociodemographic data and risk factors for overweight and obesity. Additionally, clinical data (height, weight, blood pressure) were verified. Results: The sample had a predominance of women $(n=65,60.7 \%)$ with a mean age of 16.5 years and family income of $2-4$ minimum wages $(n=53,49.5 \%)$. The prevalence of overweight and obesity was 18 (16.8\%) and $9(8.4 \%)$, respectively. It is noteworthy that 62 (58\%) individuals always skipped one meal, 54 (50.5\%) always ate while watching television, and $56(52.3 \%)$ did not do physical activity outside school. Trying and failing to go on a diet was associated with overweight and obesity, and curvilinear selfimage was associated with obesity. Conclusion: The study revealed that a significant part of the sample was overweight or obese. The failure to go on a diet and the curvilinear selfimage associated with nutritional changes suggest that the adolescents were aware of these changes and were concerned with their own weight, seeking to go on a diet in an attempt to lose weight.

Descriptors: Adolescent Nutrition; Pediatric Obesity; Overweight.
Artigo Original
1) Universidade Paulista - UNIP - São Paulo (SP) - Brasil
Recebido em: 16/02/2015 Revisado em: 28/05/2015 Aceito em: 13/07/2015 


\section{RESUMEN}

Objetivo: Verificar la prevalencia y los factores asociados con el sobrepeso y la obesidad entre adolescentes de una escuela pública de Campinas, São Paulo. Métodos: Estudio observacional y transversal realizado entre julio y septiembre de 2013 con 107 jóvenes entre 15 y 19 años que cursaban la educación secundaria en una escuela pública del municipio de Campinas, São Paulo. Se utilizó un cuestionario para investigar los datos sociodemográficos y los factores de riesgo para el sobrepeso y la obesidad. Además, se verificaron los datos clínicos (peso, altura, presión arterial). Resultados: La muestra tuvo el predominio de mujeres $(n=65$, $60,7 \%$ ) con media de 16,5 años y renta familiar entre 2 y 4 sueldos mínimos $(n=53,49,5 \%)$. La prevalencia de sobrepeso y obesidad fue de $18(16,8 \%)$ y $9(8,4 \%)$, respectivamente. Se destaca que 62 (58\%) personas siempre omitían una comida, 54 $(50,5 \%)$ de ellas siempre se alimentaba mirando la televisión y 56 (52,3\%) no practicaban actividad física fuera de la escuela. El hecho de intentar y no lograr hacer la dieta estuvo asociado con el sobrepeso y la obesidad y la autoimagen estuvo asociada con la obesidad. Conclusión: El estudio reveló que parte significativa de la muestra tenía sobrepeso u obesidad. El fallo de mantener una dieta y la autoimagen asociadas con las alteraciones nutricionales sugiere que los adolescentes tenían conciencia de estas alteraciones y se preocupaban con su propio peso hasta buscar la dieta para intentar adelgazar.

Descriptores: Nutrición del Adolescente; Obesidad Pediátrica; Sobrepeso.

\section{INTRODUÇÃO}

Atualmente, a obesidade tornou-se um problema de saúde pública, destacando-se no cenário epidemiológico mundial. Tanto países desenvolvidos como em desenvolvimento, entre eles o Brasil, possuem altos índices de pessoas acima do peso em suas populações ${ }^{(1)}$. Em função da sua magnitude e rapidez de crescimento, esse fenômeno já é considerado uma pandemia ${ }^{(2)}$.

A obesidade integra o grupo de Doenças e Agravos Não Transmissíveis (DANTs), tem caráter multifatorial e envolve questões biológicas, históricas, ecológicas, ambientais, sociais, culturais políticas e de causas desconhecidas. Vários fatores contribuem para sua prevalência, dentre eles, o atual estilo de vida da população urbana, que se traduz em hábitos alimentares não saudáveis e baixo nível de atividade física ${ }^{(2,3)}$.

No Brasil, o VIGITEL 2014, um inquérito telefônico realizado com cerca de 40.000 adultos em 26 capitais e o Distrito Federal, registrou 52,5\% de indivíduos com sobrepeso e 17,9\% com obesidade. Esses indicadores vêm crescendo anualmente e configuram uma perspectiva sombria para a saúde, uma vez que essas alterações nutricionais são sérios fatores de risco para hipertensão, diabetes, doenças cardiovasculares e câncer ${ }^{(4)}$.

No entanto, o crescimento da obesidade não atinge apenas a população adulta, uma vez que a obesidade infantil e na adolescência já se configura como um elemento de preocupação cada vez maior para a saúde pública ${ }^{(5)}$.

A adolescência é uma fase de constantes transformações biopsicossociais, tendo a nutrição um papel importante e de grande complexidade ${ }^{(3,6)}$. Evidências científicas apontam que os níveis de prevalência de sobrepeso e obesidade nos adolescentes são significativos. Estatísticas recentes do IBGE apontam que um em cada cinco jovens entre 10 e 19 anos apresenta excesso de peso ${ }^{(6)}$.

Estudo realizado com 480 adolescentes da rede pública da cidade de Juiz de Fora, Minas Gerais, detectou que a prevalência de sobrepeso foi de 7,5\% em meninas e 13,1\% em meninos; e de obesidade de $10,4 \%$ entre meninas e $7,6 \%$ entre meninos ${ }^{(7)}$.

Entre os fatores de risco para a obesidade infantil e na adolescência estão o fato de ter pais obesos, a influência dos meios de comunicação, sedentarismo, alimentação inadequada, fatores genéticos, nível socioeconômico, entre $\operatorname{outros}^{(2,8)}$.

Adolescentes com excesso de peso vêm apresentando cada vez mais problemas sérios de saúde que comprometem sua qualidade de vida. Essas complicações podem aparecer na infância e na adolescência, mas certamente estarão presentes na vida adulta ${ }^{(9,10)}$. Além disso, alterações nutricionais na adolescência podem afetar a percepção e a satisfação corporal, a autoimagem e autoestima, ocasionando distúrbios psicossociais ${ }^{(11-13)}$.

Pelo exposto, enfatiza-se a necessidade de sistemas de vigilância do estado nutricional dos adolescentes a fim de dimensionar esse fenômeno. O estudo tem como objetivo verificar a prevalência e os fatores associados ao sobrepeso e obesidade entre adolescentes de uma escola pública em Campinas, São Paulo.

\section{MÉTODOS}

Trata-se de um estudo observacional, do tipo transversal, realizado em uma unidade de ensino público da região Oeste do município de Campinas, São Paulo. A população de estudo foi composta por 107 estudantes do ensino médio, de ambos os sexos, matriculados no período matutino, entre a $1^{\mathrm{a}}$ e a $3^{\mathrm{a}}$ série, no ano de 2013.

Realizou-se a coleta de dados entre julho e setembro de 2013, por meio de um instrumento elaborado exclusivamente para esse fim. O questionário continha 17 questões objetivas sobre dados sociodemográficos (sexo, idade, renda familiar), fatores potencialmente associados 
ao sobrepeso e obesidade (percepção da própria saúde, histórico familiar de doenças crônicas, hábitos alimentares, atividade física etc.), avaliação da autoimagem corporal, e sobre dados clínicos e antropométricos (peso, altura, pressão arterial). Estes últimos aferidos no momento da coleta, utilizando-se instrumentos devidamente calibrados.

Para avaliar o estado nutricional, utilizaram-se os critérios da Organização Mundial da Saúde (OMS) ${ }^{(14)}$ para classificar o IMC, conforme a seguir: escore $\mathrm{z}$ menor que -2 foi identificado com IMC baixo; escore $z$ entre - 2 e +1 , como IMC adequado; escore $\mathrm{z}$ entre $+1 \mathrm{e}+2$, como sobrepeso; e escore $\mathrm{z}$ maior ou igual $\mathrm{a}+2$, como obesidade. A autoimagem foi apresentada em uma escala de sete níveis, composta por figuras de pessoas com diferentes formas físicas que variavam de extremamente retilínea (nível 1) a extremamente curvilínea (nível 7).

Os dados foram analisados utilizando estatística descritiva, apresentando frequência absoluta e relativa para as variáveis categóricas e média \pm desvio padrão (DP). Para as análises inferenciais, aplicou-se teste de associação $\chi^{2}$ quadrado de Pearson entre proporções, utilizando o software SPSS 13.0, com nível de significância de 5\% ( $\mathrm{p} \leq 0,05)$.

O presente estudo teve aprovação do Comitê de Ética da Universidade Paulista - UNIP Campinas $\left(\mathrm{n}^{\circ}\right.$ do protocolo 17430513.8.0000.5512), e anuência da escola e dos pais dos alunos, seguindo a Resolução 466/12 do CNS para pesquisa em seres humanos.

\section{RESULTADOS}

Entre os 107 estudantes, a idade média foi de 16,5 anos $(\mathrm{DP}=1,19)$, sendo $65(60,7 \%)$ do sexo feminino; além disso, $53(49,5 \%)$ possuíam renda familiar entre dois e quatro salários mínimos (Tabela I).

Diferença significativa entre gêneros foi observada apenas em relação à percepção da própria saúde (Tabela II), pois enquanto $33(50,8 \%)$ moças relataram que sua saúde era regular, $20(47,6 \%)$ rapazes relataram que sua saúde era $\operatorname{ruim}\left(\chi^{2}=13,568 ; \mathrm{p}<0,01\right)$.

Tabela I - Perfil sociodemográfico da amostra de adolescentes e jovens. Campinas-SP, 2013.

\begin{tabular}{lc}
\hline Variáveis Sociodemográficas & $\begin{array}{c}\text { Frequências } \\
\mathbf{n = 1 0 7}\end{array}$ \\
\hline Sexo & $\mathbf{n}(\mathbf{\%})$ \\
Feminino & $65(60,7)$ \\
Masculino & $42(39,3)$ \\
Idade (anos) & \\
Media ( \pm DP) & $16,4( \pm 1,19)$ \\
Mínimo & 15 \\
Máximo & 19 \\
Renda Familiar - salários mínimos & $\mathbf{n}(\mathbf{\%})$ \\
Até 1 & $7(6,5)$ \\
1 a 2 & $47(43,9)$ \\
2 a 3 & $27(25,2)$ \\
3 a 4 & $26(24,3)$ \\
Mais de 4 & $0(0,0)$ \\
\hline
\end{tabular}

A prevalência de sobrepeso e obesidade foi de aproximadamente $25 \%$, em conjunto $(28,6 \%$ dos homens e $23,1 \%$ das mulheres, diferença não estatisticamente significante $-\mathrm{p}=0,523)$. A maioria dos jovens apresentava pressão arterial normal $(n=55,51,4 \%)$, entretanto, parte significativa da amostra apresentava pressão limítrofe $(n=22,20,6 \%)$ ou hipertensão $(n=20,18,7 \%)$.

Com relação aos fatores de risco à saúde, $70(65,4 \%)$ dos jovens desconhecem antecedentes familiares para obesidade, 76 (71\%) desconhecem antecedentes para hipertensão e $80(74,8 \%)$ desconhecem antecedentes para diabetes. Reconhecer a obesidade, hipertensão e diabetes entre pai, mãe ou ambos foi admitido por 18 (16,8\%), 19 $(17,8 \%)$ e $16(14,9 \%)$, respectivamente.

Quanto à dieta para perder peso, 75 (70,1\%) dos participantes relataram não fazer, porém, $62(57,9 \%)$ admitiram sempre omitir uma refeição, principalmente o café da manhã $(n=32,43 \%)$. Além disso, $99(92,6 \%)$ 
Tabela II - Percepção da própria saúde e fatores de risco a saúde da amostra de adolescentes e jovens, segundo sexo. Campinas-SP, 2013.

\begin{tabular}{|c|c|c|c|c|}
\hline Percepção da Própria Saúde e Fatores de Risco à Saúde & $\begin{array}{c}\text { Masculino } \\
\mathrm{n}=\mathbf{4 2}\end{array}$ & $\begin{array}{c}\text { Feminino } \\
n=65\end{array}$ & $\begin{array}{c}\text { Total } \\
\mathbf{n}=\mathbf{1 0 7}\end{array}$ & Valor de $p$ \\
\hline Percepção da própria saúde & $\mathbf{n}(\%)$ & $\mathbf{n}(\%)$ & $\mathbf{n}(\%)$ & \\
\hline Muito boa & $0(0)$ & $5(7,7)$ & $5(4,7)$ & 0,004 \\
\hline Boa & $7(16,7)$ & $16(24,7)$ & $23(21,5)$ & \\
\hline Regular & $15(35,7)$ & $33(50,8)$ & $48(44,9)$ & \\
\hline Ruim & $20(47,6)$ & $11(16,9)$ & $31(29,0)$ & \\
\hline Muito ruim & $0(0)$ & $0(0)$ & $0(0)$ & \\
\hline IMC & n $(\%)$ & n (\%) & n (\%) & \\
\hline Desnutrido & $2(4,8)$ & $1(1,5)$ & $3(2,8)$ & 0,295 \\
\hline Normal & $28(67,7)$ & $49(75,4)$ & $77(72,0)$ & \\
\hline Sobrepeso & $7(16,7)$ & $11(16,9)$ & $18(16,8)$ & \\
\hline Obesidade & $5(11,9)$ & $4(6,2)$ & $9(8,4)$ & \\
\hline Classificação da Pressão Arterial & n $(\%)$ & n $(\%)$ & n $(\%)$ & \\
\hline Ótima & $3(7,1)$ & $7(10,8)$ & $10(9,3)$ & 0,096 \\
\hline Normal & $16(38,1)$ & $39(60,0)$ & $55(51,4)$ & \\
\hline Limítrofe & $12(28,6)$ & $10(15,4)$ & $22(20,6)$ & \\
\hline Hipertensão grau I & $10(23,8)$ & $9(13,8)$ & $19(17,8)$ & \\
\hline Hipertensão grau II & $1(2,4)$ & $0(0)$ & $1(0,9)$ & \\
\hline Uso de medicamento & n (\%) & n $(\%)$ & n $(\%)$ & \\
\hline Sim & $5(11,9)$ & $15(23,1)$ & $20(18,7)$ & 0,148 \\
\hline Não & $37(88,1)$ & $50(76,9)$ & $87(81,3)$ & \\
\hline Antecedentes familiares de obesidade & n (\%) & n (\%) & n $(\%)$ & \\
\hline Pai & $2(4,8)$ & $5(7,7)$ & $7(6,5)$ & 0,396 \\
\hline Mãe & $1(2,4)$ & $5(7,7)$ & $6(5,6)$ & \\
\hline Ambos & $3(7,1)$ & $2(3,1)$ & $5(4,7)$ & \\
\hline Desconhece & $26(61,9)$ & $44(67,7)$ & $70(65,4)$ & \\
\hline Não possui & $10(23,8)$ & $9(13,8)$ & $19(17,8)$ & \\
\hline Antecedentes familiares de hipertensão & n (\%) & n $(\%)$ & n $(\%)$ & \\
\hline Pai & $6(14,3)$ & $2(3,1)$ & $8(7,5)$ & 0,113 \\
\hline Mãe & $5(11,9)$ & $3(4,6)$ & $8(7,5)$ & \\
\hline Ambos & $1(2,4)$ & $2(3,1)$ & $3(2,8)$ & \\
\hline Desconhece & $25(59,5)$ & $51(78,5)$ & $76(71,0)$ & \\
\hline Não possui & $5(11,9)$ & $7(10,8)$ & $12(11,2)$ & \\
\hline Antecedentes familiares de diabetes & n $(\%)$ & n $(\%)$ & n $(\%)$ & \\
\hline Pai & $3(7,1)$ & $4(6,2)$ & $7(6,5)$ & 0,653 \\
\hline Mãe & $4(9,5)$ & $4(6,2)$ & $8(7,5)$ & \\
\hline Ambos & $1(2,4)$ & $0(0,0)$ & $1(0,9)$ & \\
\hline Desconhece & $29(69,0)$ & $51(78,5)$ & $80(74,8)$ & \\
\hline Não possui & $5(11,9)$ & $6(9,2)$ & $11(10,3)$ & \\
\hline Dieta para perder peso & n $(\%)$ & n $(\%)$ & n $(\%)$ & \\
\hline Sim, sempre & $4(9,5)$ & $9(13,8)$ & $13(12,1)$ & 0,750 \\
\hline Não & $31(73,8)$ & $44(67,7)$ & $75(70,1)$ & \\
\hline Tentou e não conseguiu & $7(16,7)$ & $12(18,5)$ & $19(17,8)$ & \\
\hline Omite uma refeição & n $(\%)$ & n (\%) & n $(\%)$ & \\
\hline Não, nunca & $14(33,3)$ & $18(27,7)$ & $32(29,9)$ & 0,715 \\
\hline Sim, às vezes & $4(9,5)$ & $9(13,8)$ & $13(12,1)$ & \\
\hline Sim, sempre & $24(57,1)$ & $38(58,5)$ & $62(57,9)$ & \\
\hline Qual refeição omite $(n=75)$ & n $(\%)$ & n (\%) & n (\%) & \\
\hline Café da manhã & $15(54,0)$ & $17(36,0)$ & $32(43,0)$ & 0,115 \\
\hline Almoço & $9(32,0)$ & $11(23,0)$ & $20(27,0)$ & \\
\hline jantar & $4(14,0)$ & $19(40,0)$ & $23(31,0)$ & \\
\hline TV ao se alimentar & n $(\%)$ & n $(\%)$ & n $(\%)$ & \\
\hline Nunca & $4(9,5)$ & $4(6,2)$ & $8(7,5)$ & 0,806 \\
\hline Sempre & $21(50,0)$ & $33(50,8)$ & $54(50,5)$ & \\
\hline As vezes & $17(40,5)$ & $29(43,1)$ & $45(42,1)$ & \\
\hline
\end{tabular}

$* \chi^{2}=13,568$ 
Tabela III - Nível de atividades físicas relatado pelos adolescentes, segundo sexo. Campinas-SP, 2013.

\begin{tabular}{lccc}
\hline Nível de Atividade Física & $\begin{array}{c}\text { Masculino } \\
\mathbf{n = 4 2}\end{array}$ & $\begin{array}{c}\text { Feminino } \\
\mathbf{n}=\mathbf{6 5}\end{array}$ & $\begin{array}{c}\text { Total } \\
\mathbf{n = 1 0 7}\end{array}$ \\
\hline Atividade física na escola* & $\mathbf{n} \mathbf{( \% )}$ & $\mathbf{n}(\mathbf{\%})$ & $\mathbf{n} \mathbf{( \% )}$ \\
Uma vez por semana & $8(19,0)$ & $13(20,0)$ & $21(19,6)$ \\
Duas vezes por semana & $18(42,9)$ & $13(20,0)$ & $31(29,0)$ \\
Às vezes & $16(38,1)$ & $39(60,0)$ & $55(51,4)$ \\
Atividade física fora da escola** & $\mathrm{n}(\%)$ & $\mathrm{n}(\%)$ & $\mathrm{n}(\%)$ \\
De 1 a 2 vezes por semana & $9(21,4)$ & $10(15,4)$ & $19(17,8)$ \\
De 2 a 3 vezes por semana & $3(7,1)$ & $3(4,6)$ & $6(5,6)$ \\
Mais de 3 vezes por semana & $16(38,1)$ & $10(15,4)$ & $26(24,3)$ \\
Não pratica & $14(33,3)$ & $42(64,6)$ & $56(52,3)$ \\
\hline
\end{tabular}

$* \mathrm{x}^{2}=6,994 ; \mathrm{p}=0,03$

$* * \mathrm{x}^{2}=11,002 ; \mathrm{p}=0,01$

Tabela IV - Fatores associados a alterações no estado nutricional. Campinas-SP, 2013.

\begin{tabular}{|c|c|c|c|c|c|}
\hline Fatores associados & $\begin{array}{c}\text { Sobrepeso } \\
n=18\end{array}$ & $\begin{array}{c}\text { Valor de } \\
\text { p }\end{array}$ & $\begin{array}{c}\text { Obesidade } \\
n=9\end{array}$ & $\begin{array}{c}\text { Valor de } \\
\text { p }\end{array}$ & $\begin{array}{l}\text { Total } \\
\mathbf{n}=\mathbf{2 7}\end{array}$ \\
\hline Percepção da própria saúde & n (\%) & & n (\%) & & n (\%) \\
\hline Muito boa & $2(11,1)$ & 0,254 & $0(0)$ & 0,460 & $2(7,4)$ \\
\hline Boa & $4(22,2)$ & & $2(22,2)$ & & $6(22,2)$ \\
\hline Regular & $5(27,8)$ & & $6(66,7)$ & & $11(40,7)$ \\
\hline Ruim & $7(38,9)$ & & $1(11,1)$ & & $8(29,6)$ \\
\hline Muito ruim & $\mathbf{0}(\mathbf{0})$ & & $\mathbf{0}(\mathbf{0})$ & & $\mathbf{0}(\mathbf{0})$ \\
\hline Dieta para perder peso & $\mathrm{n}(\%)$ & & $\mathrm{n}(\%)$ & & $\mathrm{n}(\%)$ \\
\hline Sim, sempre & $3(16,7)$ & $0,002 *$ & $1(11,1)$ & $0,044 * *$ & $4(14,8)$ \\
\hline Não & $7(38,9)$ & & $5(55,6)$ & & $2(44,4)$ \\
\hline Tentou e não conseguiu & $8(44,4)$ & & $3(33,3)$ & & $11(40,7)$ \\
\hline Omite uma refeição & n $(\%)$ & & n (\%) & & n (\%) \\
\hline Não, nunca & $2(11,1)$ & 0,159 & $2(22,2)$ & 0,358 & $4(14,8)$ \\
\hline Sim, às vezes & $3(16,7)$ & & $0(0)$ & & $3(11,1)$ \\
\hline Sim, sempre & $13(72,2)$ & & $7(77,8)$ & & $20(74,1)$ \\
\hline Atividade física na escola & n $(\%)$ & & n $(\%)$ & & n (\%) \\
\hline Uma vez por semana & $2(11,1)$ & 0,462 & $1(11,1)$ & 0,531 & $3(11,1)$ \\
\hline Duas vezes por semana & $7(38,9)$ & & $4(44,4)$ & & $11(40,7)$ \\
\hline Às vezes & $9(50,0)$ & & $4(44,4)$ & & $13(48,1)$ \\
\hline Atividade física fora da escola & n (\%) & & n $(\%)$ & & n (\%) \\
\hline De 1 a 2 vezes por semana & $3(16,7)$ & 0,711 & $1(11,1)$ & 0,766 & $4(14,8)$ \\
\hline De 2 a 3 vezes por semana & $0(0,0)$ & & $0(0)$ & & $0(0,0)$ \\
\hline Mais de 3 vezes por semana & $5(27,8)$ & & $3(33,3)$ & & $8(29,6)$ \\
\hline Não pratica & $10(55,6)$ & & $5(55,6)$ & & $15(55,6)$ \\
\hline TV ao se alimentar & n (\%) & & n $(\%)$ & & n (\%) \\
\hline Nunca & $1(5,6)$ & 0,611 & $0(0)$ & 0,256 & $1(3,7)$ \\
\hline Sempre & $11(61,1)$ & & $3(33,3)$ & & $14(51,9)$ \\
\hline As vezes & $6(33,3)$ & & $6(66,7)$ & & $12(44,4)$ \\
\hline Autoimagem & n $(\%)$ & $0,002 * * *$ & n $(\%)$ & & n (\%) \\
\hline 1 & $0(0)$ & & $0(0)$ & $0,0001 \#$ & $0(0)$ \\
\hline 2 & $0(0)$ & & $0(0)$ & & $0(0)$ \\
\hline 3 & $5(27,8)$ & & $0(0)$ & & $5(18,5)$ \\
\hline 4 & $5(27,8)$ & & $2(22,2)$ & & $7(25,9)$ \\
\hline 5 & $5(27,8)$ & & $0(0)$ & & $5(18,5)$ \\
\hline 6 & $2(11,1)$ & & $6(66,7)$ & & $8(29,6)$ \\
\hline 7 & $1(5,6)$ & & $1(11,1)$ & & $2(7,4)$ \\
\hline
\end{tabular}

$* \mathrm{X}^{2}=12,049 ; * * \mathrm{X}^{2}=14,344 ; * * * \mathrm{X}^{2}=20,545 ;{ }^{*} \mathrm{X}^{2}=47,814$ 
relataram comer vendo televisão, em frequências que variaram entre às vezes e sempre.

Com relação ao nível de atividades físicas, houve diferença significativa entre os gêneros quanto ao local em que foi realizada (Tabela III). Nas atividades realizadas na escola, $39(60 \%)$ das mulheres realizavam "às vezes", enquanto $18(42,9 \%)$ dos homens realizavam "duas vezes por semana" $\left(\chi^{2}=6,994 ; p=0,03\right)$. Nas atividades físicas realizadas fora da escola, enquanto $42(64,6 \%)$ mulheres não praticavam, $28(66,6 \%)$ homens praticavam de uma a mais de três vezes por semana $\left(\chi^{2}=11,002 ; \mathrm{p}=0,01\right)$.

Ao se avaliar apenas os adolescentes com sobrepeso e obesidade $(n=27), 11(40,7 \%)$ consideraram seu estado de saúde regular, e $8(29,6 \%)$ o consideraram ruim. Além disso, $11(40,7 \%)$ jovens declararam que tentaram fazer dieta e não conseguiram, enquanto $20(74,1 \%)$ assumiram sempre omitir uma refeição (Tabela IV).

Treze $(48,1 \%)$ dos jovens com sobrepeso ou obesidade relataram que praticavam "às vezes" atividade física na escola, e $15(55,6 \%)$ não praticavam fora da escola. Esse nível de inatividade é coerente com o dado de que 14 (51,9\%) sempre viam televisão ao se alimentar e com a identificação por $15(55,5 \%)$ adolescentes com uma autoimagem mais curvilínea (níveis 5, 6 e 7 da escala de autoimagem).

Apenas dois fatores foram estatisticamente associados ao sobrepeso e à obesidade: dieta para perder peso e autoimagem. Isso sugere que mais jovens com sobrepeso e obesidade tentaram e não conseguiram fazer dieta $\left(\chi^{2}=12,049 ; \mathrm{p}=0,002\right)$, e em especial os obesos estavam cientes dessas alterações nutricionais, uma vez que indicaram as figuras mais curvilíneas na escala de autoimagem $\left(\chi^{2}=47,814 ; p \leq 0,0001\right)$.

\section{DISCUSSÃO}

A saúde dos adolescentes e adultos jovens preocupa cada vez mais os órgãos e profissionais da saúde pública. Apesar de ser uma população pouco propensa a problemas de saúde, observam-se com frequência alterações no estado nutricional.

O estudo identificou que a prevalência de sobrepeso e obesidade na amostra estudada foi de aproximadamente $25 \%$ ( $16,8 \%$ de sobrepeso e $8,4 \%$ de obesidade). Além disso, também foi observada a prevalência de hipertensão em quase $20 \%$ dos jovens.

Estudos regionais apontam variações consideráveis nessas taxas. Pesquisa realizada com mais de 4.000 estudantes entre 14 e 19 anos da rede pública estadual de Pernambuco estimou prevalência de $11,5 \%$ de sobrepeso e $2,4 \%$ de obesidade ${ }^{(15)}$. Um estudo epidemiológico de base populacional realizado com 1.253 estudantes de 7 a 17 anos, matriculados em escolas da rede pública e privada, da cidade de Maceió, Alagoas, detectou a prevalência de 9,3\% de sobrepeso e $4,5 \%$ de obesidade ${ }^{(16)}$.

Prevalência de cerca de $50 \%$ de sobrepeso e obesidade foi observada em estudo desenvolvido com 217 crianças entre 7 e 11 anos de escolas da rede privada da cidade de Fortaleza-CE. Os autores ressaltam que tais índices apontam a gravidade da situação da saúde entre os escolares da cidade de Fortaleza ${ }^{(17)}$.

Essas variações, aliadas às limitações inerentes aos estudos transversais, sugerem que a comparação entre as taxas deve ser cautelosa. No entanto, é razoável supor que $25 \%$ é parte significativa da amostra e se configura como um sério agravo à saúde, principalmente se considerarmos que essas alterações clínicas estão ocorrendo em uma fase de crescimento e desenvolvimento, como a adolescência.

Quase $45 \%$ dos participantes avaliaram seu estado de saúde como regular. No entanto, os rapazes se diferenciaram das meninas, por o considerarem ruim. A maioria dos adolescentes admitiu comer vendo televisão e omitir uma refeição, isto é, possuía hábitos de vida que favorecem o excesso de peso.

Tais comportamentos estão associados ao estilo de vida moderno, no qual o desenvolvimento econômico e o processo de urbanização provocaram modificações importantes no padrão alimentar e predomínio do sedentarismo. Além disso, as comodidades oferecidas pelo mundo moderno, tais como aparelhos de televisão, telefones sem fio, videogames, computadores, controle remoto, entre outros, têm favorecido a redução do gasto energético ${ }^{(18)}$.

A omissão de refeições, principalmente do café da manhã, é outro dado relevante. Considerado uma das principais refeições, a omissão do café da manhã e a baixa frequência do consumo de leite entre crianças da Região Sul do Brasil foram significativamente associadas à obesidade ${ }^{(19)}$. É consenso entre profissionais de saúde que a prática regular de desjejum favorece o consumo de grãos, frutas e produtos lácteos, além de proporcionar um maior fracionamento das refeições ao longo do dia, fato que está inversamente ligado a hábitos alimentares, como "beliscar" alimentos com alta densidade energética durante o dia ${ }^{(20)}$.

A promoção da alimentação saudável é uma das diretrizes da Política Nacional de Alimentação e Nutrição e integra as estratégias citadas pela Política Nacional de Promoção da Saúde, compondo o eixo "promoção de hábitos saudáveis, com ênfase à alimentação saudável, atividade física, comportamentos seguros e combate ao tabagismo". Além disso, é apontada como componente fundamental na construção da Segurança Alimentar e Nutricional, aqui entendida como "a realização do direito humano a uma alimentação saudável, acessível, de qualidade, em quantidade suficiente e de modo permanente, 
sem comprometer o acesso a outras necessidades essenciais, com base em práticas alimentares saudáveis, respeitando as diversidades culturais e sendo sustentável do ponto de vista socioeconômico e agroecológico"(3,9).

A inatividade física fora da escola foi relatada por mais de $50 \%$ dos participantes, sendo as meninas mais sedentárias do que os rapazes. Atualmente, a OMS recomenda para adultos a prática de atividades físicas de intensidade moderada, com frequência de pelo menos cinco dias da semana, 30 minutos por dia, para a prevenção de doenças cardiovasculares, diabetes e alguns tipos de câncer. Essas atividades podem ser praticadas de forma contínua ou fracionadas em duas ou três vezes ao longo do dia ${ }^{(5,13)}$. No caso dos adolescentes, a recomendação é de pelo menos 45 minutos, três a quatro dias por semana ${ }^{(21,22)}$.

Níveis insuficientes de atividade física entre adolescentes estão bem documentados na literatura. Estudo realizado com 4.216 estudantes do ensino médio, com idade entre 14 e 19 anos, identificou que $65,1 \%$ eram insuficientemente ativos, isto é, indivíduos que não realizavam pelo menos 60 minutos diários de atividades físicas moderadas ou vigorosas, durante cinco ou mais dias por semana ${ }^{(19)}$.

Dados da Pesquisa Nacional de Saúde Escolar apontaram que, dos quase 61.000 jovens brasileiros entrevistados, cerca de $40 \%$ dos meninos e $60 \%$ das meninas eram insuficientemente ativos. O estudo chama atenção para a necessidade de promover estratégias para incremento de atividades físicas entre adolescentes ${ }^{(23)}$.

No grupo de adolescentes com sobrepeso e obesidade, os comportamentos de comer vendo televisão, omitir refeição e ser inativo fora da escola se repetiram. A tentativa de fazer dieta e a autoimagem curvilínea associada às alterações nutricionais sugere preocupação com o peso e a consciência de que a aparência está fora dos padrões de normalidade.

A relação entre obesidade e autoimagem negativa está bastante documentada na literatura científica. Estudo realizado com adolescentes obesos atendidos no Ambulatório do Hospital das Clínicas da Universidade Federal de Pernambuco concluiu que os adolescentes percebem a obesidade como doença, o que acarreta em baixa autoestima e sensação de isolamento ${ }^{(24)}$. Dois estudos de revisão da literatura sobre obesidade e aspectos psicossociais na adolescência apontam que a obesidade abala a autoestima e interfere na vida pessoal, gerando angústia e frustação ${ }^{(25,26)}$.

Embora exista pouco incentivo ao controle da obesidade na adolescência, é evidente que se fazem necessárias campanhas mais intensas. A escola é o local ideal para a realização de políticas de intervenção no sobrepeso e obesidade, porque a maioria dos adolescentes passa grande parte do tempo na escola. Além disso, o ambiente escolar tem influência sobre a saúde, pois as escolas fornecem aos estudantes as ferramentas necessárias para que eles entendam as orientações de saúde divulgadas por diversos meios de comunicação. Tem, ainda, um papel fundamental no desenvolvimento psicológico e emocional dos adolescentes, e pode incluir as informações atualizadas sobre saúde no currículo tradicional ou em disciplinas específicas voltada para a promoção da saúde ${ }^{(21,22,27)}$.

Portanto, é de grande importância que sejam elaborados programas educacionais inovadores, campanhas mais efetivas, visando ampliar o conhecimento dos adolescentes sobre nutrição e saúde, e que influenciem de forma positiva os hábitos alimentares e a prática de atividade física diária, garantindo melhor qualidade de vida no presente e no futuro. E, assim, garantir que a saúde comece na escola.

\section{CONCLUSÃO}

O estudo revelou que parte significativa da amostra estava com sobrepeso ou obesidade. A falha em manter uma dieta, a autoimagem curvilínea, associada às alterações nutricionais, sugere que os adolescentes tinham consciência dessas alterações e se preocupavam com o próprio peso, a ponto de buscarem a dieta para tentar emagrecer.

Além disso, foram observados comportamentos não apropriados durante a alimentação, como ver televisão e omitir uma refeição.

\section{REFERÊNCIAS}

1. Terres NG, Pinheiro RT, Horta BL, Pinheiro KAT, Horta LL. Prevalência e fatores associados ao sobrepeso e à obesidade em adolescentes. Rev Saúde Pública. 2006;40(4):627-33.

2. Barbieri AF, Mello RA. As causas da obesidade: uma análise sob a perspectiva materialista histórica. Rev Facul Educação Física Unicamp. 2012;10(1):133-53.

3. Ministério da Saúde (BR). Obesidade. Brasília: Secretaria de Atenção à Saúde; 2006. (Caderno de Atenção à Saúde, n. 12).

4. Ministério da Saúde (BR), Secretaria de Vigilância em Saúde, Departamento de Vigilância de Doenças e Agravos não Transmissíveis e Promoção da Saúde. VIGITEL - Vigilância de fatores de risco e proteção para doenças crônicas por inquérito telefônico. Brasília: Ministério da Saúde (BR); 2015.

5. Verde SMML. Obesidade infantil: o problema de saúde pública do século 21. Rev Bras Promoç Saúde. 2014;27(1):1-2. 
6. Enes CC, Slater B Obesidade na adolescência e seus principais fatores determinantes. Rev Bras Epidemiol. 2010;13(1):163-71.

7. Rodrigues PA, Marques MH, Chaves MGAM, Souza CF, Carvalho MF. Prevalência e fatores associados a sobrepeso e obesidade em escolares da rede pública. Ciênc Saúde Coletiva. 2011;16(Supl 1):1581-8.

8. Freitas ASS1, Coelho SC, Ribeiro RL. Obesidade infantil: influência de hábitos alimentares inadequados. Saúde \& Amb Rev. 2009;4(2):9-14.

9. Bergmann GG, Bergmann MLA, Moreira RB, Pinheiro ES, Marques AC, Gaya A. Sobrepeso e obesidade na infância e adolescência: possibilidades de medidas e reflexões sobre as propostas de avaliação. Rev Bras Ativ Fís Saúde. 2011;16(1):62-9.

10. Ferreira JS, Aydos RD. Prevalência de hipertensão arterial em crianças e adolescentes obesos. Ciênc Saúde Coletiva. 2010;15(1):97-104.

11. Caram LA, Lomazi EA. Hábito alimentar, estado nutricional e percepção da imagem corporal de adolescentes. Rev Adolesc Saúde. 2012;9(2):21-9.

12. Rosa MF, Gonçalves S. Moderadores e mediadores da relação entre a psicopatologia e a obesidade ou sobrepeso na adolescência. Psicol Saúde Doenças. 2011;12(2):224-36.

13. Branco LM, Hilário MOE, Cintra IP. Percepção e satisfação corporal em adolescentes e a relação com seu estado nutricional. Rev Psiquiatr Clín (São Paulo). 2006;33(6):1-7.

14. Nobre LN, Sammour SNF, Costa Sobrinho PS. Índice de massa corporal e circunferência de cintura como preditores de pressão arterial alterada em adolescentes. Rev Méd Minas Gerais. 2011;21(4):404-12.

15. Tassitano RM, Barros MVG, Tenório MCM, Bezerra J, Hallal PC. Prevalência e fatores associados ao sobrepeso e à obesidade em adolescentes, estudantes de escolas de Ensino Médio de Pernambuco, Brasil. Cad Saúde Pública. 2009;25(12):2639-52.

16. Mendonça MRT, Silva MAM, Rivera IR, Moura AA. Prevalência de sobrepeso e obesidade em adolescentes da cidade de Maceió. AMB Rev Assoc Med Bras. 2010;56(2):192-6.

17. Paula FAR, Lamboglia CMGF, Silva VTBL, Monteiro MS, Moreira AP, Pinheiro MHNP, et al. Prevalência de sobrepeso e obesidade em escolares da rede pública e particular da cidade de Fortaleza. Rev Bras Promoç Saúde. 2014;27(4):455-61.
18. Oliveira AMA, Cerqueira EMM, Souza JS, Oliveira AC. Sobrepeso e obesidade infantil: influência de fatores biológicos e ambientais em Feira de Santana, BA. Arq Bras Endocrinol Metab. 2003;47(2):144-50.

19. Tenório MCM, Barros MVG, Tassitano RM, Tenório JM, Hallal PC. Atividade física e comportamento sedentário em adolescentes estudantes do ensino médio. Rev Bras Epidemiol. 2010;13(1):105-17.

20. Sociedade Brasileira de Cardiologia: VI Consenso Brasileiro de Hipertensão. Rev Bras Hipertens. 2010(1):7-10.

21. Rivera IR, Silva MAM, Silva RATA, Oliveira BAV, Carvalho ACC. Atividade física, horas de assistência à TV e composição corporal em crianças e adolescentes. Arq Bras Cardiol. 2010;95(2):159-65.

22. Secretaria Municipal de Saúde do Estado de São Paulo. Manual de Atenção à Saúde do Adolescente. São Paulo; 2006.

23. Hallal PC, Knuth AG, Cruz DKA, Mendes MI, Malta DC. Prática de atividade física em adolescentes brasileiros. Ciênc Saúde Coletiva. 2010;15(Supl 2):3035-42.

24. Serrano SQ, Vasconcelos MGL de, Silva GAP, Cerqueira MMO, Pontes CM. Percepções do adolescente obeso sobre as repercussões da obesidade em sua saúde. Rev Esc Enferm USP. 2010;44(1):25-31

25.Santos LM, Dias MRMG, Uchimura KY. Percepções de adolescentes obesos sobre seu estado nutricional. Rev Eletrônica Facul Evang Paraná 2012;2(3):72-84.

26. Alli LR, Mattos AP, Halpern R, Bergmann MLA, Costanzi CB, Rech RR. Obesidade infantil e fatores psicossocioculturais. RBPFEX. 2007;1(3):21-8.

27. Silveira JAC, Taddei JAAC, Guerra PH, Nobre MRC. A efetividade de intervenções de educação nutricional nas escolas para prevenção e redução do ganho excessivo de peso em crianças e adolescentes: uma revisão sistemática. J Pediatr (Rio J). 2011;87(5):38292.

\section{Endereço para correspondência:}

Maria Meimei Brevidelli

Clínica de Saúde Integrada

Rua Luis Gois, 2211

Bairro: Mirandópolis

CEP: 04043-400 - São Paulo - SP - Brasil

E-mail: meibi@unip.br 